\title{
LENR IN RUSSIA
}

Anri A. Rukhadze

Prokhorov General Physics Institute, Russian Academy of Sciences, http:/ /www.gpi.ru

Moscow 119991, Russian Federation

rukh@fpl.gpi.ru

\section{Vladimir I. Grachev}

Kotel'nikov Institute of Radioengineering and Electronics, Russian Academy of Sciences, http:/ /www.cplire.ru Moscow 125009, Russian Federation

grachev@cplire.ru

Abstract. Provides information on research into low energy nuclear reactions, held in Russia. Shows a great variety of methodological approaches, which is typical for Russian studies in this area. Lists the most vivid experiments, the problem of low reproducibility of their results also provided the basic theoretical work that illustrate attempts of interpretation of the known experimental results. Formulated possible at the moment understanding low-energy nuclear reactions, collective nuclear transformations occurring at energies unacceptably low for conventional nuclear reactions, and give a variation of the isotopic composition and high heat in the absence of residual radioactivity. Keywords: cold transformation of nuclears, the reproducibility of the experimental results, the attempts of theoretical interpretation

УАК 530.145, 539.17

Bibliography -8 references

Received 20.06.2017

RENSIT, 2017, 9(1):5-7

DOI: $10.17725 /$ rensit.2017.09.005

\section{CONTENTS}

1. INTRODUCTION (5)

2. LENR IN RUSSIA (6)

3. Pubications of this issue (6)

4. Conclusion (7)

REFERENCES (7)

\section{INTRODUCTION}

From the first steps of nuclearphysics, its development has been largely, in General, in two directions - of high- and low-energetic. E. Rutherford (1871-1937), the "father" of nuclear physics who discovered (1919) artificial "transmutation of elements" - nuclei $\left({ }_{7}^{14} \mathrm{~N}+{ }_{2}^{4} \mathrm{He} \rightarrow{ }_{8}^{17} \mathrm{O}+{ }_{1}^{1} \mathrm{H}[1,2]\right)$ by fast alpha particles, have inter-preted this "modern alchemistry" as the area of high-energy processes, which opening the era of nuclear power.

Available by the time the works on nuclear transmutations of stable isotopes in natural conditions, and primarily the receipt by the american chemists K. Irion and J. Wendt (1922) of helium in alpha-decay of tungsten, induced of electric explosion [3], Rutherford declared as error [4]. Then low-energetic ("cold") transmutation of nucleuses for many years turned "pseudoscience".

Nevertheless, reports of studies of lowenergy nuclear reactions occasionally appeared. Including, among others, the famous 1956 I.V. Kurchatov lecture on thermonuclear reactions in gas discharge in Harwell (England), E.I. Zababakhin bubble thermonuclear fusion [5], as well as the world's first reactor LENR 1957 of moscow constructor I.S. Filimonenko, which generating the heat excess in the system "the palladium-deuterium", etc.

And after the publication in 1989 M. Fleischmann and S. Pons (USA) in Journal of Electroanalytical Chemistry [6] and their interview on March 23, 1989 in the newspaper "Financial Times" in which they stated that it is possible to create the energy source of the industrial scale based on the fusion of nuclei of heavy hydrogen at room temperature, such researches have widely developing in many laboratories around the world under the trenchant title "cold fusion", coined by journalists.

Are appeared generators JET-MIT "NANOR" (MIT, USA), Patterson (USA), Mayer (USA), J. 
Arata (Japan), Y. Iwamura Japan), Shoulders (USA), R. Mills USA), A. Rossi nickel-hydrogen reactor (Italy), etc.

In countries such as USA, Japan, Italy, China and other, these studies are funded by the state programs and the largest firms.

Since 1990, are conducted annual International Conferences on Cold Fusion (ICCF, since 2007 - International Conferences on Condensed Matter Nuclear Science) in the United States, Japan, Italy, France, China, Korea, India and other countries.

\section{LENR IN RUSSIA}

In Russia during these studies have included the moscow RRC "Kurchatov Institute", the dubna Joint Institute for nuclear research, sarov RFNC, novosibirsk INP SB RAS, Institute of physical chemistry RAS, etc.

The studies are used the term "low energy nuclear reactions" (LENR) and conducted at a wide variety of methodological approaches (that is typical for Russian studies in this area).

These are Parkhomov's analogies of the reactor A. Rossi, Klimov's plasma vortex reactor (JIHT RAS), Kirkinskii's deuterium heat generator (IGMSB RAS), Bazhutov's heatgeneraror of plasma electrolyte (IZMIRAN), Urutskoev's electric explosion of foil, Krymsky's nano-pulse generator, Savvatimova's glow discharge, Nigmatulin-Taleyarkhan deuterated acetone, Shafeev's laser quenching of radioactivity (IOF RAS), Kornilova's transmutation of elements in biological systems (Lomonosov MSU) and many others.

I.e. the phenomenon of low-energy nuclear reactions was discovered in various experimental facilities.

This are reactions in the crystal lattice of metals induced by electrolysis or by saturating it with hydrogen, and reactions in the crystal lattice, induced by impact, the different reactions by diffusion through the membrane. This is a whole class of experiments using electric discharge, electric explosion, ultrasound in a gas or liquid. This are reactions induced corpuscular and wave action on condensed media, and finally, reactions in biological systems.

For many works in this area is characterized by a low reproducibility of the experimental results.
This is due to the fact that while still fail to find the key parameter that controls the process, because there is no understanding of the physical model of the phenomenon and the conditions of implementation regimes that promote low-energy reactions

There is also a lot of fairly elaborate theoretical models which tend to focus on resolution of the presence of anomalously large transparency of the Coulomb barrier and practically do not consider other equally important features of low-energy nuclear reactions, such as the lack of daughters radioactive isotopes, as well as the features of concomitant radiations.

Of the currently available results it follows that low energy nuclear reactions are not synthesis or decay, but, apparently, they are some collective nuclear transformations that occur at unacceptably low energies for thermonuclear reactions, and give the variation of the isotopic composition and high heatgeneration at the absence of residual radioactivity.

\section{THE PUBLICATIONS OF THIS ISSUE}

The editorial Board of the journal "Radioelectronics. Nanosystems. Information technology" (RENSIT) provides the pages of his issue for publishing a series of review materials on the studies of low energy nuclear reactions, held in Russia.

Formed the sampling includes three theoretical overview of V.A. Andreev (Lebedev Physical Institute of RAS), of S.F. Timashev (Karpov Institute of Physical Chemistry) and V.I. Vysotsky (T.Shevchenko Kiev national university) who have been developing their approaches on the foundations of modern quantum theory and five experimental works A.A. Kornilova (Lomonosov MSU), A.G. Shafeev (Prokhorov IOF RAS), A.G. Parkhomov (OIG "K. I. T."), G.V. Mishinsky (JINR), V.V. Krymsky (UrSU), the results of which are obtained by using modern experimental and diagnostic techniques.

Under the cover of one of the issue was not possible to place equally interesting results of other authors [7]. But and done sampling gives an idea about the level and scope of Russian research in this area. 


\section{CONCLUSION}

Presented works indicate a significant advancement in the understanding of weak nuclear processes and can serve as a promising subject of fruitful discussions, the broad expert consideration of the phenomenon of nuclear reactions at low energies.

It is obvious that the mastery of low-energy nuclear reactions will allow to solve many applied tasks, including the creation of a cheap standalone energetical plants, high-performance technologies of deactivation of nuclear waste, obtaining rare isotopes and transformation of chemical elements.

\section{REFERENCES}

1. Rutherford E. Collision of $\alpha$-particles with light atoms. I. Hydrogen. II. Velocity of the hydrogen atom. III. Nitrogen and oxygen atoms. IV. An anomalous effect in nitrogen. - London, Edinburg and Dublin Philos. Mag. and J. Sci., London, 1919, ser. 6, 37, p. 537-585.

2. Rutherford E. Sovremennaya alkhimiya [Modern alchemistry]. UFN, 1938, 19(1):18-48 (in Russ.).

3. Wendt GL, Irion CE. Experimental attempts to decompose tungsten at high temperatures. J.of the American Chemical Society, 1922, 44(9):1887-1894.

4. Rutherford E. Identification of a missing element. Nature, London, 1922, 109:781.

5. Kurchatov IV. O vozmozhnosti sozdaniya termoyadernykh reactsiy $\mathrm{v}$ gazovom razryade [About possibility of creation of thermonuclear reactions in gas discharge]. UFN, 1956, 59(4):603-618.

6. Zababakhin EI, Zababakhin IE. Yavleniya neogranichennoy kumulyatsii [Phenomena of unlimited cumulation]. Moscow, Nayka Publ., 1988, 161 p.

7. Fleishmann M, Pons S, Hawkins M. Electrochemically induced nuclear fusion of deuterium. J. Electroanal. Chem., 1989, 261:301-308.

8. Erlikh GV, Andreev SN. Istoriya yadernoy fiziki v zerkale alkhimii [The history of nuclear physics in the mirror of alchemistry]. Moscow, Lenand Publ., 2017, 232 p. 